\title{
Human immunodeficiency virus type 1 infection alters enzymatic and ultrastructural features of peripheral blood monocytes
}

\author{
Ali M. Gabali, Tarek Jazaerly, Ronald Cleveland ${ }^{1}$, Lawrence Kass ${ }^{1}$
}

Department of Pathology, Detroit Medical Center, Wayne State University, 3990 John R, Detroit, MI, 48201, ${ }^{1}$ Department of Pathology, Metro Health Medical Center, Case Western Reserve University, Cleveland, Ohio, USA

\begin{tabular}{|c|}
\hline Access this article online \\
\hline Website: www.avicennajmed.com \\
\hline DOI: 10.4103/2231-0770.112787 \\
\hline Quick Response Code: \\
$\square$ \\
\hline
\end{tabular}

ABSTRACT
INTRODUCTION: Human immunodeficiency virus-1 (HIV-1) infected monocytes are now
believed to serve as a reservoir for HIV-1 infection, and to play a role in viral rebound phenomena
in certain groups of patients who failed or stopped highly active antiretroviral therapy (HAART).
Data characterizing the morphological changes of peripheral blood monocytes in HIV-1-infected
individuals are limited. MATERIALS AND METHODS: In this study, we collected monocytes
from 21 asymptomatic HIV-1-infected individuals with CD4 count more than 500 cells/mm ${ }^{3}$ and
healthy individuals. The monocytes ultrastructural morphologic changes and $\alpha$-naphthyl butyrate
esterase (ANBE) activity were compared between the two groups. RESULTS: In monocytes
from patients infected with HIV-1, activity of $\alpha$-naphthyl butyrate esterase (ANBE) was markedly
increased compared with normal monocytes. In both light microscopic and ultrastructural studies,
the cytoplasm of monocytes from HIV-1-infected patients contained a haphazard appearing
network of thin fibrils. Cell surface expression of the activation marker HLA-DR molecule was
upregulated. There were no discernible differences between the cell surface expression of CD4,
CD14, and CD16 molecules comparing normal monocytes to those from HIV-1-infected patients.
CONCLUSIONS: Possibly, changes in the activity of ANBE along with a disrupted appearing
cytoplasmic fibril network contribute to monocyte dysfunction in HIV-1-infected patients.
Key words: $\alpha$-naphthyl butyrate esterase, CD16, CD14, CD4, human immunodeficiency
virus-1, monocyte

\section{INTRODUCTION}

Previous studies have shown that human immunodeficiency virus-1 (HIV-1) can infect peripheral blood monocytes without producing cytopathic effects, and replication-competent HIV-1 has been identified in monocytes. ${ }^{[1]}$ Thus, HIV-1-infected monocytes are now believed to serve as a reservoir for HIV-1 infection, and to play a role in viral rebound phenomena in certain groups of patients who failed or stopped highly active antiretroviral therapy (HAART). ${ }^{[2]}$ Recent publications have studied the association between HIV-1 infection and the expansion or loss of certain monocyte subtypes, monocyte function and chemokine production. Monocytes obtained from HIV-1-infected patients showed an increase in the number of CD14+/CD16+ monocyte subset and impaired cytotoxic activity and chemotaxis. ${ }^{[3-6]}$ To date, data characterizing the morphological changes of peripheral blood monocytes in HIV-1-infected individuals are scarce. In our study, we evaluated peripheral blood monocytes from patients infected with HIV-1. We found that they have ultrastructural morphologic abnormalities and enzyme abnormalities not found in monocytes from normal individuals.

\section{MATERIALS AND METHODS}

\section{Source of samples}

The Institutional Review Board at Metro Health Medical Center at Case Western Reserve University approved the 
study protocol. Peripheral venous blood samples obtained from asymptomatic $21 \mathrm{HIV} 1$-infected individuals with CD4 count more than 500 cells $/ \mathrm{mm}^{3}$ and were used in this study regardless of viral load. All Patients were on HAART. As controls, blood samples from $6 \mathrm{HIV}$-1-negative age-matched individuals were studied concurrently. Because, the aim of the study was to evaluate the morphologic changes in monocytes in specific time interval using blood samples obtained from individuals living in specific geographic area, our control samples satisfy the case-control study base principle.

\section{Reagents and antibodies}

Fluorescein isothiocyanate (FITC)-conjugated mouse IgG2b anti-human CD14, Phycoerythrin (PE)-conjugated mouse IgG1 anti-human CD16, PE-conjugated mouse IgG2a anti-human HLA-DR, Peridinin Chlorophyll Protein (PerCP)-conjugated mouse IgG1 anti-human CD4, FITC-conjugated mouse IgG2b, PE-conjugated mouse IgG1, PE-conjugated mouse IgG2a, and PerCP-conjugated mouse IgG1 were purchased from BD Biosciences (San Jose, CA). FACS lysing solution for whole blood was obtained from Becton Dickinson (San Jose, CA). Ficoll-Hypaque was purchased from Whittaker M. A. Bioproducts (Walkersville, $\mathrm{MD})$. Millonig's phosphate buffer solution was obtained from EM Sciences (Gibbstown, NJ). Osmium tetroxide and glutaraldehyde were obtained from Polysciences, Inc. (Warrington, PA).

Peripheral blood mononuclear cells isolation and wright staining

PBMC were isolated from the venous blood of HIV-1 patients or healthy adult individuals by density gradient centrifugation as described previously. ${ }^{[7]}$ Isolated mononuclear cells were washed twice with Hank's balanced salt solution (HBSS), $\mathrm{pH}$ 7.4. $\mathrm{PBMC}$ smears were stained with Wright stain using an automated slidemaker-stainer contained in a Sysmex XE-2100 in the Core Laboratory Facility in the Department of Pathology at Metro Health Medical Center.

\section{$\alpha$-naphthyl butyrate esterase activity}

ANBE (also known as non-specific esterase) membrane reactivity is found in monocytes and macrophages. The reaction is specific for identifying monocytes by light microscopy as it does not present on cell surface of other cell lineages. However, lymphocytes may show some punctate cytoplasmic reactivity of ANBE with no membranous pattern observed. ${ }^{[8]}$ To measure the ANBE activity, PBMC suspended in HBSS were cytocentrifuged at $\times 300 \mathrm{~g}$ onto glass slides for $1 \mathrm{~min}$ with high acceleration (Shandon, Scientific Products, Astmoor, England). Cytochemical staining for ANBE of the cytospin smears was performed as described previously. ${ }^{[7]}$ For both normal monocytes and those from HIV-1-infected patients, 100 monocytes from each person were compared and evaluated by two independent observers. ANBE results we analyzed first by tabulating the positive cells on Microsoft Office Excel 2003 spreadsheet (Microsoft Corporation). After sorting by the percentage of positive cells, the ANBE positive cases at different cut-off percentages with the highest sensitivity or specificity were identified as $>30 \%$ of the monocyte fraction.

\section{Flow cytometry}

The cell surface phenotype of monocytes isolated from HIV-1-infected patients was evaluated using three-color flow cytometry analysis after labeling with monoclonal antibodies against CD4, lipopolysaccharide receptor (CD14), Fc $\gamma$ receptor III (CD16), and HLA-DR human class II MHC antigen (class II major histocompatibility complex antigen). Briefly, $100 \mu \mathrm{l}$ of whole blood obtained from HIV-1-infected patients or healthy individuals were incubated with $0.25 \mu \mathrm{g}$ FITC-conjugated mouse IgG2b anti-human CD14, PE-conjugated mouse IgG1 anti-human CD16, PE-conjugated mouse IgG2a anti-human HLA-DR, PerCP-conjugated mouse IgG1 anti-human CD4, or with isotype control antibodies for $15 \mathrm{~min}$ at room temperature in the dark. Cells were further processed using a standard lysed whole blood procedure. ${ }^{[7]}$ In this procedure, $2 \mathrm{ml}$ FACS lysing solution was added to the mixture, vortexed gently, and then incubated for $10 \mathrm{~min}$ at room temperature. The cells were centrifuged at $\times 400 \mathrm{~g}$ for $5 \mathrm{~min}$ at room temperature, washed twice in cold phosphate buffered saline and the pellets resuspended in $0.4 \mathrm{ml}$ of $0.5 \%$ paraformaldehyde. Using a FAC Scan flow cytometer in standard manufacturer-provided configuration (Becton Dickinson, San Jose, CA), the fluorescence channels FL-1, $\mathrm{FL}^{-2}$, and $\mathrm{FL}^{-3}$ were set to detect the fluorescence intensity of FITC-conjugated, PE-conjugated, and PerCP-conjugated antibodies, respectively. Prior to analysis, compensation was adjusted using control cells labeled separately with FITC-conjugated anti-CD14 and PE-conjugated anti-CD16 and PerCP-conjugated anti-CD4. The fluorescence intensity of co-expression of three markers of 10,000 cells in each sample was analyzed after gating on the monocyte population using CD14 expression and light-scatter pattern.

\section{Electron microscopy}

Specimens were processed for ultrastructural studies in the Department of Pathology at Metro Health Medical Center. Peripheral venous blood samples were obtained in a tube containing EDTA and centrifuged at room temperature at $\times 400 \mathrm{~g}$ for $20 \mathrm{~min}$. For the study, $2 \mathrm{ml}$ whole blood was used from each patient. After removing the plasma, an equal volume of $2.5 \%$ phosphate-buffered glutaraldehyde 
was added without disturbing the buffy coat and incubated for $30 \mathrm{~min}$ at room temperature. The clotted buffy coat was removed using wood applicator sticks, cut into small pieces, and then kept in a vial of fresh $0.5 \%$ phosphate-buffered glutaraldehyde for $90 \mathrm{~min}$ at room temperature. The specimens were washed twice using Millonig's phosphate buffer $0.2 \mathrm{M}$ ( $\mathrm{pH}$ 7.4) solution before fixing in $1 \%$ osmium tetroxide in $0.2 \mathrm{M}$ (pH 7.4) Millonig's phosphate buffer for $1 \mathrm{~h}$ at room temperature. After dehydration in graded alcohol and infiltration in graded Spurr (Spurr's Kit, EM Sciences), specimens were embedded in Spurr low-viscosity embedded media according to the manufacturer's protocol for standard firm hardness (EM Sciences, Gibbstown, NJ).

Ultrathin $(78 \mathrm{~nm})$ sections were cut using a RMC $\mathrm{mt}$ XL Microtome mounted on 100 mesh copper grids, and then stained with uranyl acetate in methanol and lead citrate. A Transmission Electron Microscope FEI Tecnai T12 (FEI Company, Hillsboro, OR) was used to examine the grids according to standard protocol used at the electron microscopy facility in the Department of Pathology at Metro Health Medical Center.

\section{Statistical analysis}

Statistical analysis was performed using Student's paired $t$-test. A $P<0.05$ was accepted as statistically significant.

\section{RESULTS}

On Wright-stained specimens, monocytes from normal subjects showed lavender colored cytoplasm with scattered pink granules [Figure 1a]. In contrast, monocytes from HIV-1-infected patients showed pale blue cytoplasm and contained scattered pink granules [Figure 1b]. Monocytes from normal persons contained innumerable coarse appearing cytoplasmic fibrils that were oriented parallel to each other, and crisscrossed each other resembling a

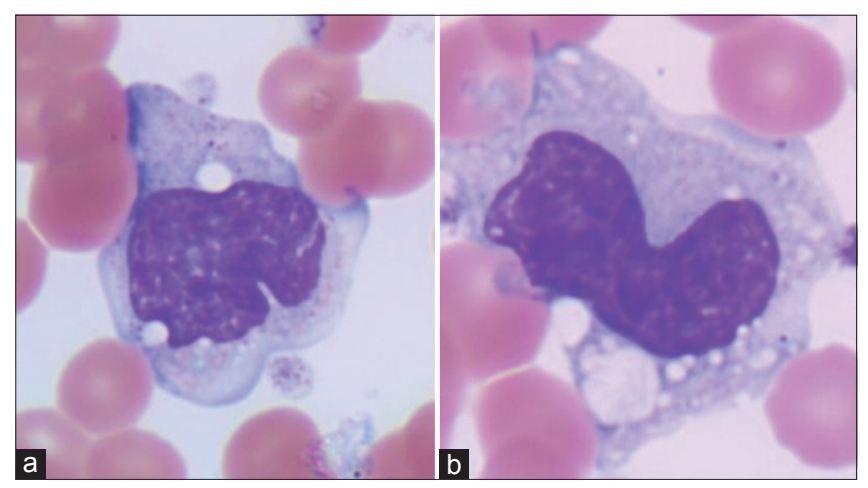

Figure 1: Wright-stained specimens, (a) monocytes from normal subjects showed lavender colored cytoplasm with scattered pink granules, coarse appearing cytoplasmic fibrils that were oriented parallel. (b) Monocytes from human immunodeficiency virus-1-infected persons showed pale blue cytoplasm and showed thin fibrils that intersected each other meshwork. Monocytes from HIV-1-infected individuals showed thin fibrils that intersected each other in a haphazard way and formed a disorderly-appearing network. The data in Table 1 summarize the light microscopy findings in both groups.

Our ultrastructural examination demonstrated a significant correlation between light microscopic findings and ultrastructural evaluation of monocytes in HIV-1-infected patients. Ultrastructurally, monocytes from normal persons showed electron dense fibrils that appeared coarse, often parallel to each other and sometimes forming a meshwork of intersecting fibrils, which did not intersect the nuclear membrane. Monocytes from HIV-1 infected patients contained innumerable thin fibrils with low electron density that appeared disorganized rather than parallel to each other. In some instances, these fibrils intersected perpendicularly into the nuclear membrane [Figure 2]. No intact virion could be recognized within the cytoplasm of monocytes from HIV-1-infected patients. Furthermore, comparing monocytes from normal persons and infected patients,

\begin{tabular}{|c|c|c|}
\hline $\begin{array}{l}\text { Table I: Comparison } \\
\text { monocytes obtained } \\
\text { healthy individuals by } \\
\text { presented as the prec } \\
\text { changes for all sampl } \\
\text { individual }\end{array}$ & $\begin{array}{l}\text { norphology of } \\
\text { n HIV-I-infecte } \\
\text { t microscopy. } \\
\text { inant cytoplasr } \\
\text { each using PBM }\end{array}$ & $\begin{array}{l}\text { ipheral blood } \\
\text { atients and } \\
\text { sults are } \\
\text { and nuclear } \\
\text { f a different }\end{array}$ \\
\hline $\begin{array}{l}\text { Cytoplasmic and } \\
\text { nuclear morphology }\end{array}$ & HIV-I negative & HIV-I positive \\
\hline Stain intensity of cytoplasm & Dense staining & Faint staining \\
\hline Cytoplasmic vacuoles & No change & No change \\
\hline Cytoplasmic granules & No change & No changes \\
\hline Cytoplasmic fibril & Course & Thin \\
\hline Meshwork & Parallel & Haphazard \\
\hline Nuclear cytoplasmic ratio & No changes & No changes \\
\hline Chromatin strands & Coarse & Thin \\
\hline Chromatin aggregates & Slightly increased & Slightly decreased \\
\hline
\end{tabular}

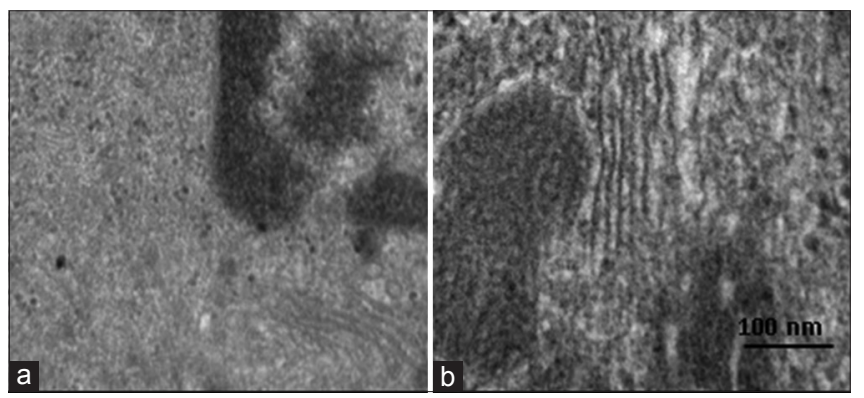

Figure 2: Electron micrographs of monocytes obtained from (a) Human immunodeficiency virus-1 (HIV-1)-infected patient and (b) healthy individual. The fibrils appear thin with low electron density and haphazard and disorganized in monocytes isolated from HIV-1-infected patient (a). Monocytes isolated from a normal individual have electron dense fibrils that appeared coarse and parallel to each other (b). The depicted images are a representative of a single experiment. Similar findings were obtained in additional experiments, each using monocytes from a different individual. Direct magnification is $\times 150000$ 
there were no recognizable morphologic differences in cell membranes like shrinking or blebbing, nuclear changes like condensation and fragmentation suggestive of apoptosis, or cytoplasmic organelles or granules.

As shown in Figure 3a, monocytes obtained from HIV-1 infected individuals demonstrated strong cytoplasmic staining for ANBE. In contrast, monocytes obtained from HIV-1-negative individuals demonstrated weak staining for ANBE [Figure 3b]. Differences in the ANBE activity between the two groups were statistically significant, namely, $66.67 \pm 16.30 \%$ cells in HIV-1-positive samples versus $5.33 \pm 1.63 \%$ cells in the control group $(P<0.0001$, $t$-test).

Results of dual-parameter dot plots of a representative experiment are shown in Figure 4. Flow measurement of the light scatter characteristics of the cells and the percentage of cells positive for CD14 monocyte marker demonstrated that the evaluated cells were free of contaminated granulocytes and lymphocytes. Results obtained from flow measurement of monocytes obtained from $21 \mathrm{HIV}-1$-positive patients and 6 HIV-1-negative individuals are shown in Figure 5. There was no significant statistical difference in the percentages of the dual expression of CD14/CD16 [Figure 5a], CD4/ CD14 [Figure 5b], CD4/CD16 [Figure 5c], HLA-DR/ CD14 [Figure 5d], and HLA-DR/CD4 [Figure 5e] cell surface markers in the monocytes of HIV-1-infected patients and those of normal control samples. The CD14+/ CD16- phenotype was significantly higher (>70\%) than CD14+/CD16+ (10\%), CD14-/CD16- (12\%), and CD14-/ $\mathrm{CD} 16+(8 \%)$ phenotypes in both of the tested monocyte populations [Figure 5a].

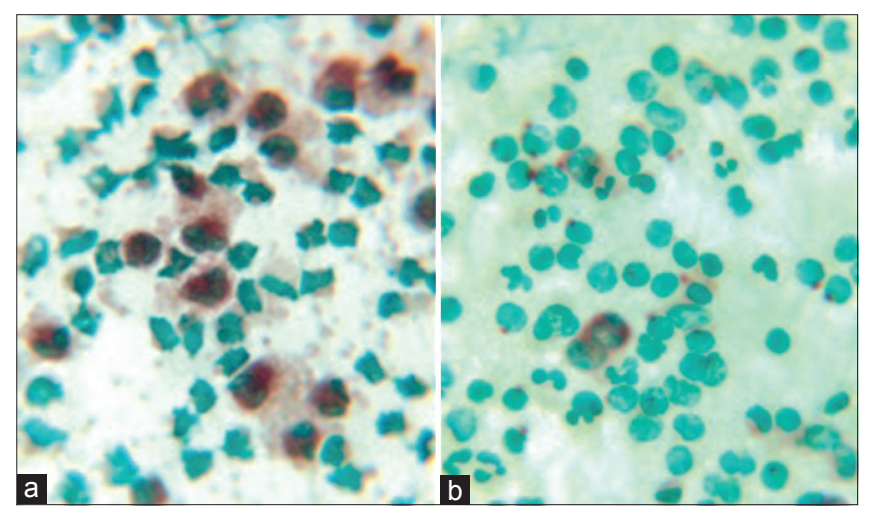

Figure 3: Light microscopy imaging of ANBE activity of monocytes obtained from (a) Human immunodeficiency virus-1 (HIV-1)-infected patient and (b) healthy individual. Monocytes in HIV-1-infected patients (a) display significantly increased ANBE activity compared with normal controls (b). The depicted images are a representative of a single experiment. Similar findings were obtained in additional experiments, each comparing monocytes from a different individual. Original magnification is $\times 40$. The light scatter characteristics of the cells that are positive for the CD14 specific monocyte marker demonstrated that the evaluated cells were free of contaminated granulocytes and lymphocytes in both samples
In contrast to the normal low CD16 expression (8\%) on CD14+ monocytes, approximately $45 \%$ of CD $14+$ monocytes expressed the CD4 molecule [Figure 5b]. The percentage of CD4 expression was slightly increased in CD14+ monocytes of HIV-1-infected patients [Figure 5b] than normal group. However, the difference was not statically significant. Another monocyte subset showing an increase in the absolute number but not statically significant is the CD4-/CD14+ phenotype in the normal control group [Figure 5b]. The CD4-/CD14- and CD4+/CD14- phenotypes formed a low percentage of the measured monocytes in both tested groups.

Dual expression of CD4/CD16 and HLA-DR/CD14 demonstrated low intensity of CD16 expression and high intensity of HLA-DR markers in both groups [Figure $5 c$ and d]. Furthermore, CD16 expression showed an inverse correlation with the CD14 but not CD4 expression in both groups [Figure $5 \mathrm{a}$ and $\mathrm{c}$ ]. Monocytes isolated from both groups showed the same level of HLA-DR expression [Figure $5 \mathrm{~d}$ and e]. In both groups, the intensity of HLA-DR expression correlated proportionally with CD14 expression [Figure 5d] and was independent of $\mathrm{CD} 4$ expression [Figure 5e]. Cells that have $\mathrm{CD} 4+/ \mathrm{CD} 16+$, CD4-/CD16+, HLA-DR-/CD14-, HLA-DR+/CD14-, HLA-DR-/CD14+, HLA-DR-/CD4-, and HLA-DR-/CD4+ phenotypes formed low percentages of the measured monocytes. Finally, there was a decrease in the absolute number of monocytes showing the HLA-DR+/ CD14- phenotype in HIV-1-infected patients compared with normal controls.

\section{DISCUSSION}

In our studies, monocytes obtained from HIV-1-infected patients display significant light microscopic abnormalities in Wright-stained smears, including pale cytoplasmic staining compared with monocytes from normal persons [Table 1]. Possibly, the decreased cytoplasmic staining intensity may be due in part to decreased stainable basic protein in monocytes from HIV-1-infected patients. Both normal monocytes and those from infected patients showed approximately the same number of cytoplasmic vacuoles and granules [Table 1]. Accordingly, it is unlikely that changes in the number of these cytoplasmic organelles contribute to the staining features seen in monocytes from infected patients.

Ultrastructurally, abnormalities in cytoplasmic fibrils and in the pattern of these fibrils appeared to correlate with, what we found in light microscopic abnormalities seen in this study. They extend the findings described in an earlier light microscopic study. ${ }^{[9]}$ In monocytes from HIV-1-infected patients, cytoplasmic fibrils were unusually thin and 

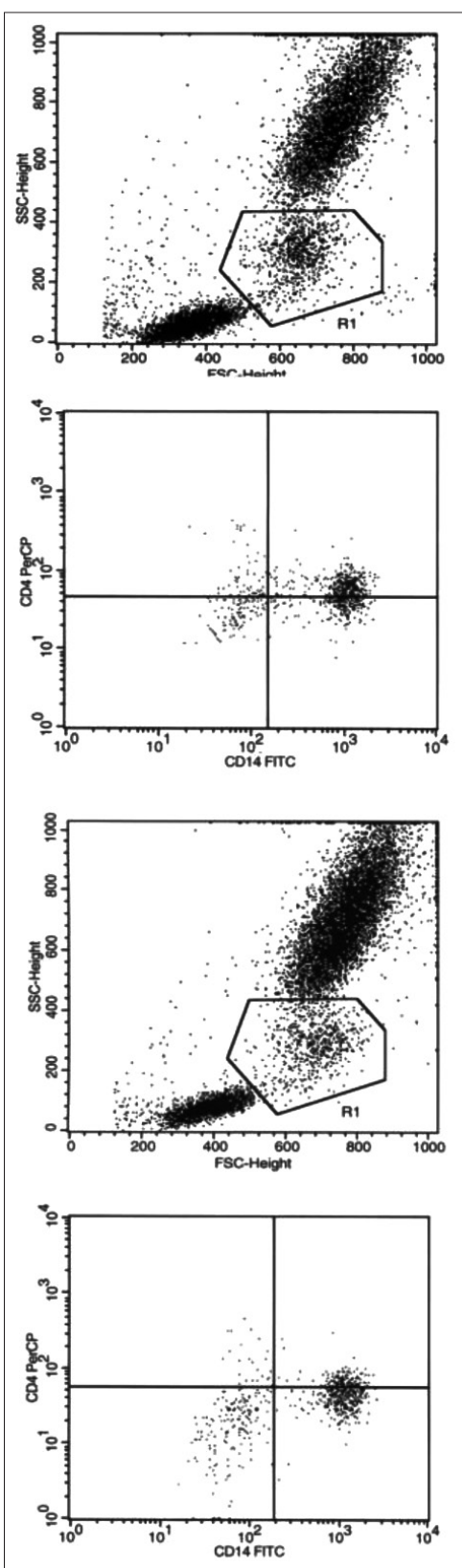
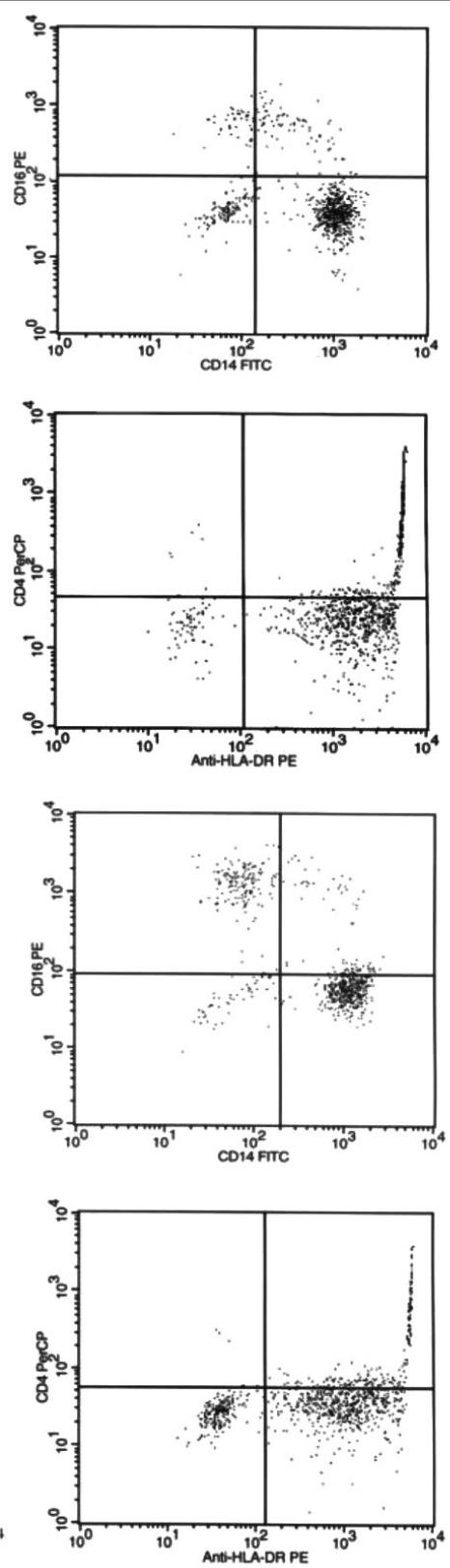
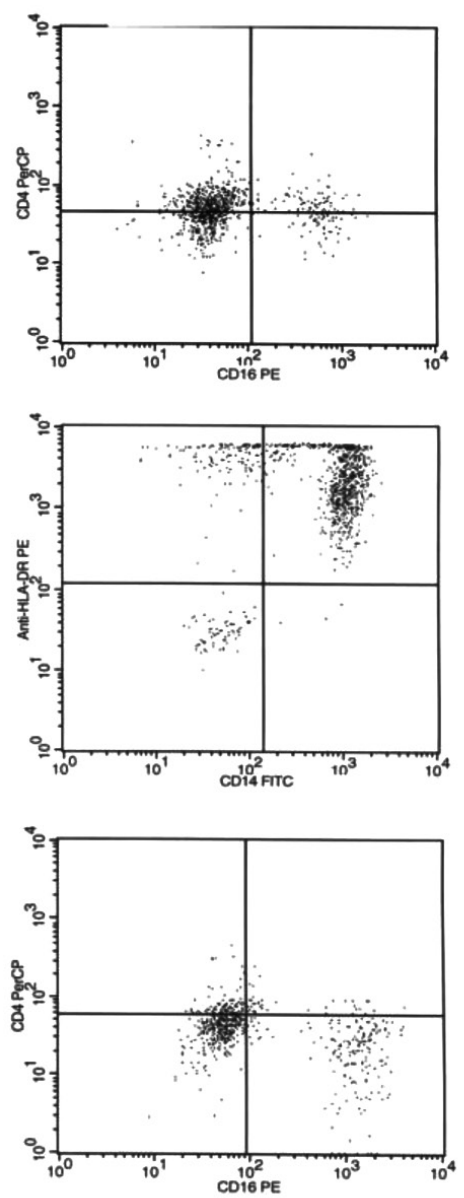

B

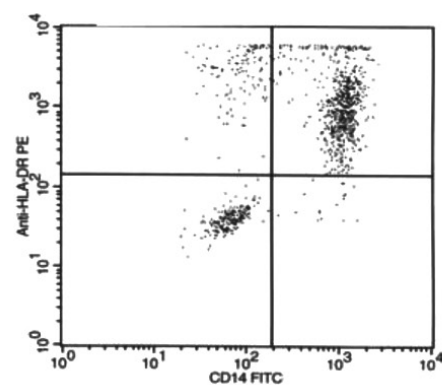

Figure 4: Panels $A$ and $B$ show a representative forward light scatter and side light scatter profile and the expression level of CD4, CD14, CD16, and HLA-DR on monocytes obtained from (A) Human immunodeficiency virus-1 infected patient and (B) normal individual. All results are from the same experiment. Similar findings were obtained in additional experiments, each comparing monocytes from a different individual

seemed to crisscross each other at various angles creating a haphazard pattern. The disorganized appearing fibrils sometimes intersected perpendicularly into the nuclear membrane. In normal monocytes, the fibrils were thick, and ran parallel to each other and to the nuclear membrane, and did not show perpendicular intersection with the nuclear membrane. Explanations for these abnormalities are unknown, but in some way may contribute to or reflect monocyte dysfunction in HIV-1-infected patients.

Previous studies have shown that HIV-1 enters monocytes by phagocytosis (CD4 independent) and CD4-dependent endocytosis, and that the CD4-mediated pathway is the infectious route of entry. ${ }^{[9,10]}$ However, no virion particles were identified within the endosomes of monocytes in HIV-1-infected patients. Although, the exact mechanism that causes such disorganized fibrillary patterns remains unknown, we speculate that they may represent the results of HIV-1 entrance or infection of the monocytes.

The results indicate monocytes in HIV-1-infected patients display significantly increased ANBE activity compared with normal controls [Figure $3 \mathrm{a}$ and $\mathrm{b}$ ]. Although, the exact function of ANBE is still unknown, studies have 


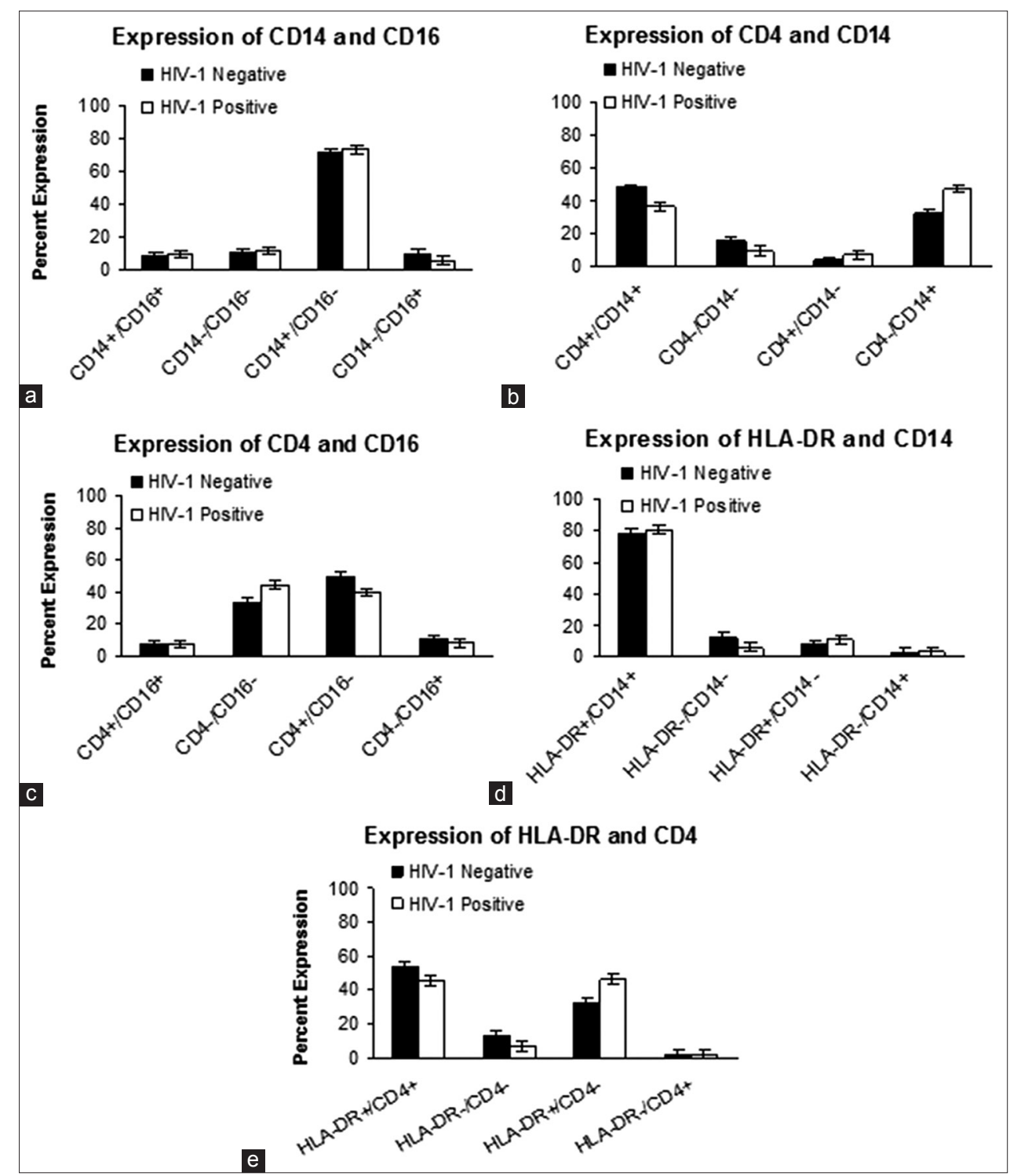

Figure 5: There was no significant statistical difference in the percentages of the dual expression of (a) CD14/CD16, (b) CD4/CD14, (c) CD4/CD16, (d) HLA-DR/ CD14, and (e) HLA-DR/CD4 cell surface markers in the monocytes of Human immunodeficiency virus-1 (HIV-1)-infected patients and those of normal control samples. Results are presented as the means \pm SE for monocytes of $21 \mathrm{HIV}$-1-infected patients and $6 \mathrm{HIV}$-1-negative healthy individual

suggested that it may play a role in the chemotactic properties of monocytes. ${ }^{[6]}$ While we have no explanation yet for this finding, it could contribute to the impaired chemotactic activity, as several reports have shown that monocytes in HIV-1-infected patients display various functional abnormalities including defective chemotaxis. ${ }^{[6]}$ The mechanism and the significance of increased ANBE activity in monocytes in HIV-1-infected individuals remain to be established.

We gated only on monocytes that were free of contaminating lymphocytes or granulocytes, as demonstrated by light scatter characteristics and the expression of CD14 monocyte markers [Figure 4]. CD14 is a lipopolysaccharide receptor that is expressed in high levels on the surface of blood monocytes of HIV-1-patient. ${ }^{[4]}$ Figure 5 demonstrates no significant difference between the expression of CD4, CD14, CD16, and HLA-DR cell surface molecules in HIV-1-infected patients and those in normal controls. However, there was a slight decrease in the absolute number of cells with dual expression of CD4+/CD14+ in HIV-1-infected patients when compared to normal controls [Figure 5b]. In contrast, the CD4-/CD14+ monocytes in the HIV-1 group showed an increase in the absolute number than the control samples.

Previous reports have shown that only a small percentage of blood monocytes are infected by HIV-1, and that those of the CD4+/CD16+ phenotype are more susceptible to HIV-1 than CD4+/CD16- phenotype subset. ${ }^{[4]}$ Interestingly, our data indicated that monocytes in HIV-1-infected 
patients do not show a preferential significant increase in CD16 expression when compared with the normal control group [Figure 5c]. In fact, CD16+ monocytes formed a small percentage $(<10 \%)$ of peripheral blood monocytes in both of the tested monocyte populations. Results from our tested group suggest that HIV-1 infection does not alter CD16 cell surface expression and subsequently may not significantly affect the phenotypic CD16-maturation of monocytes.

Monocytes play an important role in the immune response as antigen-presenting cells. HLA-DR is involved in the antigen-presenting process and T-cell activation. It is believed that HLA-DR is up-regulated in activated monocytes. The reported data of the HLA-DR role in HIV-1 infection is conflicting. Some studies have shown that HIV-1 infection of monocytes is associated with a decrease in HLA-DR mRNA transcription and HLA-DR cell surface expression. ${ }^{[11,12]}$ Other report suggests that monocytes display an increased level of HLA-DR. ${ }^{[13]}$ and that, in fact, this increase may contribute to CD4 + T-cell depletion during the course of HIV-1 infection. Our data demonstrate that HIV-1 infection does not produce a significant increase in the level of HLA-DR expression in monocytes [Figure $5 \mathrm{~d}$ and $\mathrm{e}$ ]. Together, these results suggest that HIV-1 infection produces significant morphological and cytochemical changes in peripheral blood monocytes that may potentially contribute to the pathogenesis of HIV-1 infection.

\section{REFERENCES}

1. Gorry PR, Sonza S, Kedzierska K, Crowe SM. Isolation of human immunodeficiency virus type 1 from peripheral blood monocytes. Methods Mol Biol 2005;304:25-33.

2. Sonza S, Mutimer HP, Oelrichs R, Jardine D, Harvey K, Dunne A, et al.
Monocytes harbour replication-competent, non-latent HIV-1 in patients on highly active antiretroviral therapy. AIDS 2001;15:17-22.

3. Thieblemont N, Weiss L, Sadeghi HM, Estcourt C, Haeffner-Cavaillon N. CD14lowCD16high: A cytokine-producing monocyte subset which expands during human immunodeficiency virus infection. Eur J Immunol 1995;25:3418-24.

4. Nockher WA, Bergmann L, Scherberich JE. Increased soluble CD14 serum levels and altered CD14 expression of peripheral blood monocytes in HIV-infected patients. Clin Exp Immunol 1994;98:369-74.

5. Kedzierska K, Crowe SM. The role of monocytes and macrophages in the pathogenesis of HIV-1 infection. Curr Med Chem 2002;9:1893-903.

6. Flø RW, Naess A, Nilsen A, Harthug S, Solberg CO. A longitudinal study of phagocyte function in HIV-infected patients. AIDS 1994;8:771-7.

7. Kampalath B, Cleveland RP, Chang CC, Kass L. Monocytes with altered phenotypes in posttrauma patients. Arch Pathol Lab Med 2003;127:1580-5.

8. Bozdech MJ, Bainton DF. Identification of alpha-naphthyl butyrate esterase as a plasma membrane ectoenzyme of monocytes and as a discrete intracellular membrane-bounded organelle in lymphocytes. J Exp Med 1981;153:182-95.

9. Filice G, Cereda PM, Orsolini P, Soldini L, Razzini E, Campisi D, et al. Endocytosis constitute the infectious route of HIV-1 entry in human and rabbit monocytes lacking the CD4 receptor. Microbiologica 1991;14:77-93.

10. Bedinger P, Moriarty A, von Borstel RC $2^{\text {nd }}$, Donovan NJ, Steimer KS, Littman DR. Internalization of the human immunodeficiency virus does not require the cytoplasmic domain of CD4. Nature 1988;334:162-5.

11. Shao L, Sperber K. Impaired regulation of HLA-DR expression in human immunodeficiency virus-infected monocytes. Clin Diagn Lab Immunol 2002;9:739-46.

12. Polyak S, Chen H, Hirsch D, George I, Hershberg R, Sperber K. Impaired class II expression and antigen uptake in monocytic cells after HIV-1 infection. J Immunol 1997;159:2177-88.

13. Koethe SM, Carrigan DR, Turner PA. Increased density of HLA-DR antigen on monocytes of patients infected with the human immunodeficiency virus. J Med Virol 1989;29:82-7.

Cite this article as: Gabali AM, Jazaerly T, Cleveland R, Kass L. Human immunodeficiency virus type 1 infection alters enzymatic and ultrastructural features of peripheral blood monocytes. Avicenna J Med 2013;3:1-7.

Source of Support: Nil, Conflict of Interest: None declared. 